\title{
Burnout among Physicians in Egypt during COVID-19 Pandemic
}

Doaa I. Omar ${ }^{1}$, Basma M. Hani ${ }^{1}$, Eman E. Abd-Ellatif ${ }^{2}$

${ }^{1}$ Department of Community Medicine, Faculty of Medicine, Benha University, ${ }^{2}$ Department of Community

Medicine, Faculty of Medicine, Mansoura University, Egypt

*Corresponding author: Basma M. Hani, Mobile: (+20) 1061821325, Email: basma.hany@fmed.bu.edu.eg

\begin{abstract}
Introduction: Physician burnout is considered a great public health problem, and the occurrence of the COVID-19 pandemic made it worse. The pandemic put several physical and emotional stressors on the frontline healthcare providers leading to increased burnout.
\end{abstract}

Aim of work: was to assess the prevalence of burnout and its predictors among frontline physicians in Egypt during COVID 19 pandemic and evaluating the relation between burnout, its subscales, and psychosomatic symptoms.

Materials and Methods: This descriptive cross-sectional study assessed 503 Egyptian physicians for burnout between the $26^{\text {th }}$ of March 2020 to the $1^{\text {st }}$ of June 2020, during the COVID-19 pandemic weeks, using the online form of Maslach Burnout Inventory [MBI].

Results: $72.8 \%$ of study subjects were classified as having a high burnout level and $26.0 \%$ as having an average level. $62.2 \%$ and $62.4 \%$ of studied physicians had high emotional exhaustion and depersonalization levels, respectively, and $43.5 \%$ had low personal achievement levels. Dealing with critical cases, dealing with suspected cases of COVID 19, the number of shifts per month, and the presence of psychosomatic disorders were the significant positive predictors for total burnout score while dealing with critical cases, the number of shifts per month, and the presence of psychosomatic disorders were the significant positive predictors for emotional exhaustion. Significantly higher scores of psychosomatic disorders were observed among physicians with a high level of burnout, emotional exhaustion, and depersonalization subscales.

Conclusion: It could be concluded that dealing with critical cases, suspected and confirmed cases of COVID 19 were significant positive predictors for total burnout score. The high levels of overall burnout and its subscales indicate the need for specific intervention programs to cope with the work-related stressors.

Keywords: burnout -COVID-19- physicians- Egypt- pandemic.

\section{INTRODUCTION}

By the end of 2019, the COVID-19 illness (COVID-19) was distinguished in China, especially in Whang City, in Hubei territory. In March 2020, COVID-19 was pronounced as a pandemic of general wellbeing worry because of the quick and overall spread of the sickness ${ }^{(\mathbf{1})}$.

Toward the beginning of the SARS-CoV-2 spread, medical facilities had restricted accessibility for personal protective equipment (PPE) and guidelines or therapy were not evident. Therefore, numerous experts felt befuddled and ill-equipped to treat sufficiently COVID-19 patients ${ }^{(2)}$.

During the pandemic, doctors needed to work extended periods, which led to a lack of sleep. Large numbers of them felt a loss of independence as many doctors needed to sign an agreement that alleged they could be sent any place, similar to they were in the military ${ }^{(3)}$.

On account of expanding COVID-19 patients, clinical doctors who were not worked in the irresistible infection field and who didn't have sufficient experience to treat similar conditions assembled to help the frontline physicians, which made them enduring more noteworthy pressing factors and stress when confronting COVID-19 patients ${ }^{(3)}$.

The monetary strain was another issue all through COVID-19 pandemic; as declared by the
Medical Group Management Association (MGMA), $90 \%$ of medical services suppliers have not been seeing COVID-19 patients, and for them, they suffered a great drop in their patient volume in a range of $75 \%$ to $90 \%$. The surgeons suffered great monetary strain as they were forced to postpone all non-emergency operations ${ }^{(4)}$.

The pressure of expanded working hours, Insufficient eating, no rest, deficient insurance, the dread of tainting their families, fearing of death and seeing COVID-19 patients pass on regardless of what they do, the absence of respect by hospital managers, and the dread of being terminated, prompted some enthusiastic and mental sentiments among physicians as an outrage, nervousness, and misery and influenced their psychological and passionate prosperity, prompting burnout indications ${ }^{(5)}$.

Burnout condition (BS) is "a generally common work-related issue which has been viewed as a pandemic of present-day culture that requires expanding consideration and further contemplating around the world " (6).

Burnout is a syndrome defined as "serious emotional exhaustion with poor work adaptation owing to prolonged occupational stress. It has three main components: emotional exhaustion (EE), depersonalization (DP), and diminished professional achievement (PA)" ${ }^{(7)}$. 
EE is depicted by energy lack or feeling of depletion and the absence of motivation and is brought about by the personal conflict between relationships and a substantial workload. DP is a psychological state of emotional detachment wherein people's impersonal treatment in the workplace can lead to a lazy disposition, selfish behavior, alienation, anxiety, irritability, and demotivation. Decreased PA is characterized by the propensity of a worker to negative self-assessment, resulting in them feeling less competent and successful and therefore being dissatisfied with their $\mathrm{PA}^{(\mathbf{8})}$.

As SARS-CoV-2 keeps on spreading universally, an expanded concern has been given to the forefront of medical care providers' prosperity. Internationally, before the end of November 2020, over sixty million affirmed COVID-19 cases, and more than a million and a half deaths have been accounted for by the World Health Organization ${ }^{(9)}$.

Coronavirus pandemic is certainly viewed as a long-distance race. On the off chance that we exhaust the medical care group running in the main mile of the long-distance race, the excess miles will be more earnestly. Along these lines, to keep on conveying the ideal medical services for its length, we should support our labor force and attempting to diminish burnout among them from the beginning ${ }^{(3)}$.

The aim of the current work was to assess the prevalence of burnout among physicians in Egypt during the COVID 19 pandemic and to identify associated factors and predictors for burnout syndrome subscales. In addition to evaluate the relation between burnout subscales and psychosomatic symptoms.

\section{MATERIALS AND METHODS}

Study design: A cross-sectional, descriptive study was conducted between the $26^{\text {th }}$ of March 2020 to the $1^{\text {st }}$ of June 2020, during the COVID-19 pandemic weeks. It was designed to assess the prevalence and factors linked to burnout among frontline physicians working in all health facilities in 20 governorates in Egypt. Physicians were asked to participate in the study through a series of electronic communications. An electronic version of the structured, selfadministered questionnaire distributed to them through social media (WhatsApp and Facebook medical groups and private accounts). individuals refusing participation were excluded.

The sample size was calculated considering a level of confidence of $95 \%$, expected prevalence of $53.9 \%$, and precision of 0.05 and was found to be 381 . For better accuracy and validity and to cover any losses due to non-responders, 503 physicians were included in the study.

\section{Study tool:}

Data of the study were collected using a predesigned electronic, self-administered questionnaire which included: (a) Sociodemographic, Occupational, and health-related characteristics. (b) Psychosomatic disorders symptoms. (c) burnout assessment tool; Maslach Burnout Inventory.

\section{Sociodemographic, Occupational, and health-related variables:}

Anonymous data on sociodemographic variables (age, gender, educational level, marital status, residence, family size) were collected to identify the determinants of burnout; work characteristics and experiences (job type, department or ward type, location, years of experience, number of shifts per month, frequency of dealing with critical cases, frequency of dealing with suspected COVID19 cases, frequency of dealing with confirmed COVID19 cases and private work outside the hospital); smoking history and presence of chronic health problems .

Psychosomatic disorders symptoms scale: included the following self-reported seven symptoms: lowerback pain, tension headache, sleeping problems, chronic fatigue, stomach upset, tension diarrhea, and heart palpitation. Respondents were asked: "during the past 12 months, how often have you had a back-pain?" Responses were coded as often (3), sometimes (2), seldom (1), and never (0) ${ }^{(\mathbf{1 0})}$.

The Maslach Burnout Inventory (MBI) was first reported in 1996 by Maslach $\boldsymbol{e t}$ al., and it has been considered the gold standard that is almost universally accepted for assessment of burnout due to its high validity and reliability $(\mathbf{1 1}, \mathbf{1 2})$.

Twenty-two items with a 7-point Likert scale are included in the MBI. Items are written about personal feelings or behaviors as claims. At each point, the frequency scale is defined, ranging from 0 (never) to 6 (every day). The overall cumulative burnout scale score is 132. There are three subscales to the MBI: emotional exhaustion, which represents both physical and mental exhaustion (Q no. 1-9); depersonalization, which refers to changes in the attitude of the individual when coming in contact with those who receive services as beginning to display a cold and impersonal contact with suffering (Q no. 10-14); and reduced professional accomplishment, which evaluates the perspective of the impact of others, work-related wellbeing, the relation with problems, and evidencing a feeling of dissatisfaction (Q no. 15-22).

Burnout is considered a continuous variable, categorized into low, average, and high likelihood of experiencing feelings of each burnout element. High scores on the emotional exhaustion and depersonalization subscales (maximum score of 54, 30 respectively) and low scores on the reduced professional accomplishment subscale (maximum score of 48) represent a high degree of burnout. Subscales of burnout were categorized into low, average, and high burnout levels (Table I).

Regarding the total burnout scale, scores of 133 are considered as low, $34-66$ as average, and 6799.9 as a high level of burnout. A participant was considered to meet burnout criteria if he/she got a 
"high" score on at least 2 of the three dimensions of MBI ${ }^{(11,12)}$.

Table I: Classification of scores on subscales of the Maslach Burnout Inventory (MBI) ${ }^{(11)}$. MBI subscales Level of burnout

\begin{tabular}{|l|c|c|c|}
\hline $\begin{array}{l}\text { Emotional exhaustion } \\
\text { score }\end{array}$ & $<16$ & $17-26$ & $>27$ \\
\hline $\begin{array}{l}\text { Depersonalization } \\
\text { score }\end{array}$ & $<6$ & $7-12$ & $>13$ \\
\hline $\begin{array}{l}\text { Reduced professional } \\
\text { accomplishment score }\end{array}$ & $>39$ & $38-32$ & $<31$ \\
\hline
\end{tabular}

Ethical considerations:

Approval of Benha Faculty of Medicine's Ethics committee, Benha University, was obtained with approval code (Rc.5.7.2020). An informed written, electronic consent was obtained from all participating physicians after clarifying the study's objectives, data confidentiality, and voluntary involvement.

\section{Statistical analysis}

Data were collected, refined, and coded then analyzed using SPSS software, version 25. Numerical data were presented as mean \pm standard deviation (SD). Student t-test was used to compare two groups, ANOVA test was used to compare more than two groups, correlation and linear regression were also used. Categorical data were presented as number and percent. The adopted level of significance was 0.05 at a $95 \%$ level of confidence. Data were presented in tables and graphs as appropriate.

\section{RESULTS}

\section{Levels of burnout among the studied group.}

Most of the study participants [72.8\%] were classified as having a high burnout level and $26.0 \%$ as having average burnout while $1.6 \%$ of low burnout level, according to the total burnout score. More than half $[62.2 \%, 62.4 \%]$ of studied physicians had high emotional exhaustion and depersonalization levels, respectively. Slightly less than half [43.5\%] had low personal achievement levels (Table 1).

Table [1]: Degree of Maslach burnout inventory scores among the study group (n-503)

\begin{tabular}{|c|c|c|c|c|}
\hline \multirow{2}{*}{ Variable } & \multirow{2}{*}{$\begin{array}{c}\text { Mean } \\
(\text { SD })\end{array}$} & \multicolumn{3}{|c|}{ Categorization of MBI } \\
\cline { 3 - 6 } & & $\begin{array}{l}\text { low } \\
\mathrm{n}(\%)\end{array}$ & $\begin{array}{l}\text { moderate } \\
\mathrm{n}(\%)\end{array}$ & $\begin{array}{l}\text { high } \\
\mathbf{n}(\%)\end{array}$ \\
\hline \multirow{2}{*}{$\mathrm{EE}$} & 33.7 & 70 & 120 & $\mathbf{3 1 3}$ \\
& -13 & -13.9 & -23.9 & $\mathbf{- 6 2 . 2}$ \\
\hline \multirow{2}{*}{$\mathrm{DP}$} & 14 & 60 & 129 & $\mathbf{3 1 4}$ \\
& -7.1 & -11.9 & -25.6 & $\mathbf{- 6 2 . 4}$ \\
\hline \multirow{2}{*}{ PA } & 31.6 & 107 & 177 & $\mathbf{2 1 9}$ \\
& -8.7 & -21.3 & -35.2 & $\mathbf{- 4 3 . 5}$ \\
\hline Total burnout & 79.4 & 6 & 131 & $\mathbf{3 6 6}$ \\
score & -20.4 & -1.2 & -26 & $\mathbf{- 7 2 . 8}$ \\
\hline
\end{tabular}

$\mathrm{SD}=$ standard deviation; $\mathrm{n}=$ number of individuals;

$\mathrm{EE}=$ Emotional Exhaustion; $\mathrm{DP}=$ Depersonalization;

$\mathrm{PA}=$ Personal Accomplishment

\section{Demographic data}

This study was carried out on 503 COVID19 frontline Egyptian physicians who were mostly [57.9\%] in the age group [30-39] years. More than half were females [52.7\%]. The majority of participants were currently married [67.8\%]. More than two-thirds were urbans [73.4\%]. A total of $71.4 \%$ had a family size $\geq 4$. Most participants [39.8\%] were holding a master's degree regarding the level of education (Table 2).

Occupational and health related characteristics

More than one-third [39.2\%] were consultants, $70.4 \%$ had work experience $\leq$ ten years, $23.3 \%$ were surgeons, $56.1 \%$ were working at secondary health facilities [hospitals], while $9.7 \%$ were working in isolation health facilities. $50.1 \%$ had a private work besides their basic governmental ones. The majority [70.4\%] had been worked less than ten shifts per month. $28.2 \%$ of physicians dealt with critically ill patients once a week, while those who dealt with suspected cases of COVID 19 once daily were $24.9 \%$. About half of participants [40\%] dealt with confirmed cases of COVID 19. $88.7 \%$ of the respondents were non-smokers, and $82.1 \%$ had no chronic diseases (Table 3 ).

\section{Relationship between total /sub-domains of burnout score and sociodemographic, occupational and health related factors.}

The total burnout level was significantly higher among physicians with a diploma or master's degree of education [ $\mathrm{p}=0.04]$, those dealing with confirmed COV-19 [ $p<0.001]$. Physicians with a higher number of shifts per month $[\geq 10]$, or dealing with critically ill patients, or dealing with suspected cases of COVID 19, showed a significant difference in terms of the total burnout scores $[p<0.001]$ (Table 2,3).

Higher scores of emotional exhaustions [higher burnout] were significantly observed among diploma and master's degrees of education [ $p=0.005]$. specialists [ $p=0.004]$, lower years of work experience [ $<10$ year] $[p=0.002]$, higher number of shifts [ $>10][p<0.001]$, frequent dealing with critical, suspected, and confirmed cases of COVID 19 (Table 2,3).

Concerning depersonalization, significantly higher scores were observed among younger generations of physicians $[p<0.001]$, those who had a Bachelor of Medicine $[p<0.001]$, smaller family size $[<4$ members] $[p<0.004]$, specialists $[p<0.001]$, those had lower years of work experience [ $<10$ year] $[p=0.002]$, number of shifts [ $>10][p<0.001]$, frequent dealing with critical, suspected and confirmed cases of COVID 19 (Table 2,3). 
Lower personal accomplishment scores [higher burnout] were significantly observed among the younger age group $[p<0.001]$, those who had a Bachelor of Medicine $[\mathrm{p}=0.01]$, specialists $[p<0.001]$, lower years of work experience [ $\mathrm{p}=0.001]$ and significantly higher scores of personal accomplishments were observed among physicians frequently dealing with suspected cases of COVID 19 (Table 2,3).
The significant differences in burnout and its subdomains [personal accomplishment, emotional exhaustion, and depersonalization] concerning sociodemographic multi-categorical variables were examined by ANOVA post hoc Tukey test. Since physicians who were dealing with critical or suspected cases of COVID-19, once daily or more than once daily, had higher total burnout levels compared with others [Tukey post hoc test, $p \leq 0.05]$.

Table [2]: Relation between study physicians' demographic characteristics and the three subscales of MBI (n-503).

\begin{tabular}{|c|c|c|c|c|}
\hline \multirow[t]{2}{*}{ Variables } & \multirow{2}{*}{$\begin{array}{l}\mathrm{n}(\%) \\
\text { Total }=\mathbf{5 0 3}\end{array}$} & \multicolumn{3}{|c|}{ Mean (SD) of sub scales of MBI } \\
\hline & & $\mathbf{E E}$ & DP & PA \\
\hline \multicolumn{5}{|l|}{ Age groups [years] } \\
\hline \multirow{4}{*}{$\begin{array}{l}20-29 \\
30-39 \\
40-49 \\
50 \text { and above }\end{array}$} & $\begin{array}{l}167 \\
(33.2)\end{array}$ & $35.5(12.7)$ & $15.6(7.3) * *$ & $30.1(8.9) * *$ \\
\hline & 291(57.9) & $32.7(12.8)$ & $13.4(6.8) * *$ & $31.8(8.5) * *$ \\
\hline & $35(7)$ & $32.3(14.8)$ & $11.6(6.4) * *$ & $36.6(6.1) * *$ \\
\hline & $10(2)$ & $35.9(12.8)$ & $12.1(6.6) * *$ & $\begin{array}{l}29.3(11.1) \\
* *\end{array}$ \\
\hline \multicolumn{5}{|l|}{ Educational level } \\
\hline \multirow{4}{*}{$\begin{array}{l}\text { Bachelor of medicine } \\
\text { Diploma } \\
\text { Master degree } \\
\text { Doctorate degree }\end{array}$} & $155(30.8)$ & $\begin{array}{l}34.6(12.6) \\
*\end{array}$ & $15.1(7.1) * *$ & $30.1(8.8) *$ \\
\hline & $36(7.2)$ & \multirow{2}{*}{$\begin{array}{l}34.8(12.9) \\
*\end{array}$} & \multirow[t]{2}{*}{$14.4(7.1) * *$} & \multirow[t]{2}{*}{$31.6(8.4) *$} \\
\hline & $200(39.8)$ & & & \\
\hline & $112(22.3)$ & $\begin{array}{l}30.1(13.1) \\
*\end{array}$ & $11.6(6.5) * *$ & $33.4(8.2) *$ \\
\hline \multicolumn{5}{|l|}{ Family size } \\
\hline \multirow{2}{*}{$\begin{array}{l}<4 \\
\geq 4\end{array}$} & $144(28.6)$ & $80.7(22.8)$ & $15.4(7.6) * *$ & $30.3(8.7) *$ \\
\hline & $359(71.4)$ & $78.8(19.2)$ & $13.4(6.7) * *$ & $32.09(8.6) *$ \\
\hline \multicolumn{5}{|l|}{ Smoking } \\
\hline \multirow{3}{*}{$\begin{array}{l}\text { Current Smoker } \\
\text { Ex-smoker } \\
\text { Non-smoker }\end{array}$} & $46(9.1)$ & $36.7(12.2)$ & $16.7(6.4) *$ & $31.1(8.4)$ \\
\hline & $11(2.2)$ & $28.09(14.4)$ & $11.5(10.2) *$ & $32.9(9.2)$ \\
\hline & $446(88.7)$ & $33.5(12.9)$ & $13.8(7.01) *$ & $31.6(8.7)$ \\
\hline
\end{tabular}

$\mathrm{EE}=$ Emotional Exhaustion; $\mathrm{DP}=$ Depersonalization; $\mathrm{PA}=$ Personal Accomplishment $\mathrm{SD}=$ standard deviation; $* \mathrm{P}<0.01 ; * * \mathrm{P}<0.001$ (Student's $\mathrm{t}$ test used for 2 variable and ANOVA test for more than 2 variables) 
Table [3]: Relation between occupational and health-related factors of study physicians and the three subscales of MBI (n-503).

\begin{tabular}{|c|c|c|c|c|}
\hline \multirow[t]{2}{*}{ Variable } & \multirow{2}{*}{$\begin{array}{l}\mathrm{n}(\%) \\
\text { Total }=\mathbf{5 0 3}\end{array}$} & \multicolumn{3}{|c|}{ Mean (SD) of sub scales of MBI } \\
\hline & & EE & DP & PA \\
\hline \multicolumn{5}{|l|}{ Occupational degree } \\
\hline \multirow{4}{*}{$\begin{array}{l}\text { Resident } \\
\text { Specialist } \\
\text { Consultant } \\
\text { General practitioner }\end{array}$} & $69(13.7)$ & $34.4(11.8) * *$ & $14.7(6.8) * *$ & $30.1(9.02) * *$ \\
\hline & $140(27.8)$ & $36.4(13.1) * *$ & $15.8(7.2) * *$ & $29.3(8.2) * *$ \\
\hline & 197(39.2) & $33.2(12.7) * *$ & $13.6(6.9) * *$ & $32.8(8.6) * *$ \\
\hline & $97(19.3)$ & $30.3(13.3) * *$ & $11.6(6.6) * *$ & $33.2(8.5) * *$ \\
\hline \multicolumn{5}{|l|}{ Type of the health facility } \\
\hline \multirow{3}{*}{$\begin{array}{l}\text { Primary health facility } \\
\text { Secondary health facility } \\
\text { Isolation facility }\end{array}$} & $172(34.2)$ & & & \\
\hline & $282(56.1)$ & & & \\
\hline & $49(9.7)$ & & & \\
\hline \multicolumn{5}{|l|}{ Type of the ward } \\
\hline \multirow{8}{*}{$\begin{array}{l}\text { Emergency } \\
\text { Intensive care } \\
\text { Internal medicine } \\
\text { Family medicine } \\
\text { General practice } \\
\text { Clinical pathology } \\
\text { Surgeons } \\
\text { Others }\end{array}$} & $25(5)$ & & & \\
\hline & $44(8.7)$ & & & \\
\hline & $57(11.3)$ & & & \\
\hline & $10(2)$ & & & \\
\hline & $46(9.1)$ & & & \\
\hline & $12(2.4)$ & & & \\
\hline & $117(23.3)$ & & & \\
\hline & $192(38.2)$ & & & \\
\hline \multicolumn{5}{|c|}{ Private work outside the health facility } \\
\hline Yes & $252(50.1)$ & & & \\
\hline No & 251(49.9) & & & \\
\hline \multicolumn{5}{|c|}{ The number of shifts per month } \\
\hline \multirow{2}{*}{$\begin{array}{l}\leq 10 \\
>10\end{array}$} & $354(70.4)$ & $32.5(12.9) * *$ & $13.4(6.8) * *$ & $31(6.8)$ \\
\hline & $149(29.6)$ & $36.6(12.5) * *$ & $15.5(7.4) * *$ & $32.5(8.4)$ \\
\hline \multicolumn{5}{|l|}{ Years of work experience } \\
\hline \multirow{3}{*}{$\begin{array}{l}<10 \\
10-20 \\
20+\end{array}$} & 297(59) & $35.3(12.7) * *$ & $15.3(7.1) * *$ & $30.4(8.6) * *$ \\
\hline & $206(41)$ & $31.1(12.8) * *$ & $12.4(6.6) * *$ & $33.04(8.4) * *$ \\
\hline & & $31.4(14.6) * *$ & $10(6.5) * *$ & $35.4(9.1) * *$ \\
\hline \multicolumn{5}{|c|}{$\begin{array}{l}\text { Dealing with critical patients' } \\
\text { frequency }\end{array}$} \\
\hline \multirow{6}{*}{$\begin{array}{l}\text { Never } \\
\text { Few times a year } \\
\text { Once monthly } \\
\text { Once weekly } \\
\text { Once-daily } \\
\text { More than once daily }\end{array}$} & $41(8.2)$ & $27.4(15.4) * *$ & $11.8(7.7) * *$ & $31.3(9.5)$ \\
\hline & $85(16.9)$ & $29.1(13.3) * *$ & $12.8(7.6) * *$ & $30.3(8.4)$ \\
\hline & $47(9.3)$ & $29.9(11.6) * *$ & $12.6(5.9) * *$ & $29.7(8.8)$ \\
\hline & $142(28.2)$ & $33.3(11.6) * *$ & $13.1(6.4) * *$ & $31.3(8.1)$ \\
\hline & $64(12.7)$ & $38.2(10.7) * *$ & $15.5(6.4) * *$ & $34(8.4)$ \\
\hline & $124(24.7)$ & $38.6(12.4) * *$ & $16.3(7.2) * *$ & $32.2(9.1)$ \\
\hline \multicolumn{5}{|c|}{ Frequency of dealing with suspected cases of COVID 19.} \\
\hline \multirow{4}{*}{$\begin{array}{l}\text { Never } \\
\text { Once weekly } \\
\text { Once-daily } \\
\text { More than once daily }\end{array}$} & $134(26.6)$ & $29.2(13.2) * *$ & $12.3(6.9) * *$ & $30.9(8.8) *$ \\
\hline & $165(32.8)$ & $31.4(12.2) * *$ & $12.8(6.7) * *$ & $30.2(8.6) *$ \\
\hline & $79(15.7)$ & $39(11.4) * *$ & $16.2(6.6) * *$ & $32.4(7.6) *$ \\
\hline & $125(24.9)$ & $38.1(12.2) * *$ & $16.1(7.2) * *$ & $33.4(9.03) *$ \\
\hline \multicolumn{5}{|c|}{ Dealing with confirmed cases of COVID 19} \\
\hline Yes & 201(40) & $36.7(12.2) * *$ & $15.2(6.6) * *$ & $30.9(8.8)$ \\
\hline No & $302(60)$ & $31.7(13.1) * *$ & $13.2(7.2)^{* *}$ & $31.8(8.6)$ \\
\hline
\end{tabular}

$\mathrm{EE}=$ Emotional Exhaustion; DP $=$ Depersonalization; $\mathrm{PA}=$ Personal Accomplishment $\mathrm{SD}=$ standard deviation; $* \mathrm{P}<0.01 ; * * \mathrm{P}<0.001$ (Student's t test used for 2 variable and ANOVA test for more than 2 variables)

Regarding the relation between psychosomatic score and burnout levels and its subscales, significantly higher scores of psychosomatic disorders were observed among physicians with high levels of burnout, emotional exhaustion, and depersonalization subscales (Table 4). 
Table [4]: Relation between total burnout and the three subscales of MBI among COVID-19 frontline physicians at Egypt and psychosomatic disorders (n-503)

\begin{tabular}{|c|c|c|c|c|}
\hline \multicolumn{2}{|l|}{ Variables } & \multicolumn{3}{|c|}{ Psychosomatic score } \\
\hline & & Mean \pm SD & $\mathbf{F}$ & $\mathrm{P}$ \\
\hline \multirow{3}{*}{$\begin{array}{l}\text { Total burnout } \\
\text { score }\end{array}$} & low & $10.3 \pm 4.3$ & \multirow[t]{3}{*}{23.6} & \multirow[t]{3}{*}{$<0.001 * *$} \\
\hline & moderate & $13.09 \pm 3.7$ & & \\
\hline & High & $13.3 \pm 4.2$ & & \\
\hline \multirow{3}{*}{$\begin{array}{l}\text { Emotional } \\
\text { exhaustion } \\
\text { subscale }\end{array}$} & low & $8.8 \pm 3.96$ & \multirow[t]{3}{*}{42.804} & \multirow[t]{3}{*}{$<0.001 *$} \\
\hline & moderate & $11.7 \pm 3.93$ & & \\
\hline & High & $13.4 \pm 3.68$ & & \\
\hline \multirow{3}{*}{$\begin{array}{l}\text { Depersonalization } \\
\text { subscale }\end{array}$} & low & $9.5 \pm 4.6$ & \multirow[t]{3}{*}{22.361} & \multirow[t]{3}{*}{$<0.001 *$} \\
\hline & moderate & $11.9 \pm 3.9$ & & \\
\hline & high & $13.1 \pm 3.7$ & & \\
\hline
\end{tabular}

** Highly significant

Regarding psychosomatic symptoms [low back pain, headache, sleep disorders, fatigue, tension, diarrhea, stomach upset, and palpitation] among the studied group, more than a third of participants reported sometimes suffering from these symptoms, while more than $10 \%$ reported often. Figure (1) shows that the most frequent symptoms were low back pain, headache, sleep disorders, and fatigue.

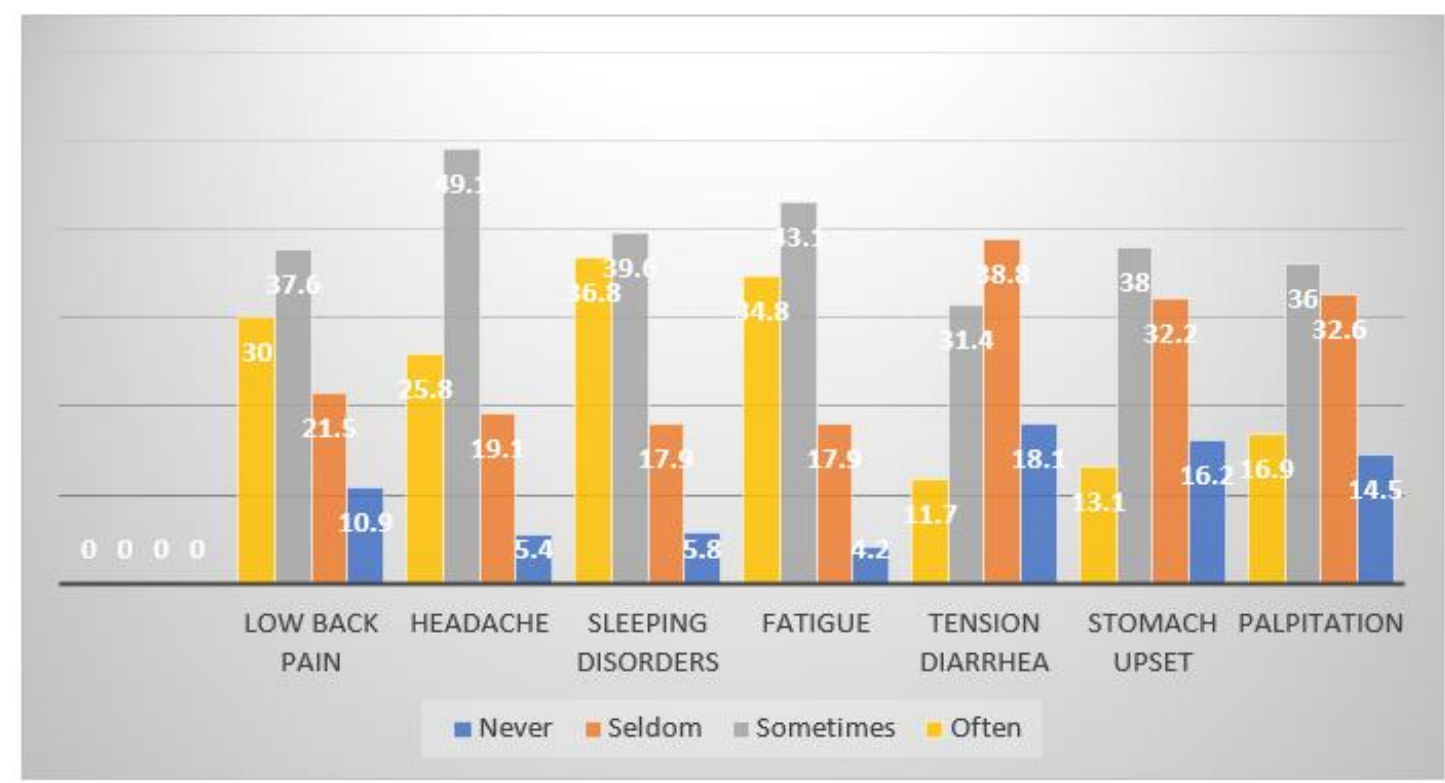

Figure (1): Distribution of psychosomatic disorders among the studied groups

\section{Correlation}

Figure $(2,3)$ showed that there is significant strong positive correlation between total burnout scale in relation with emotional and depersonalization scales [ $\mathrm{r}=0.88$ and 0.76 respectively, $\mathrm{p}<0.001]$. Moreover, significant moderate positive correlation between total burnout scale in relation with psychosomatic score and personal accomplishment score [ $\mathrm{r}=0.32$ and 0.39 respectively, $\mathrm{p}<0.001]$ 


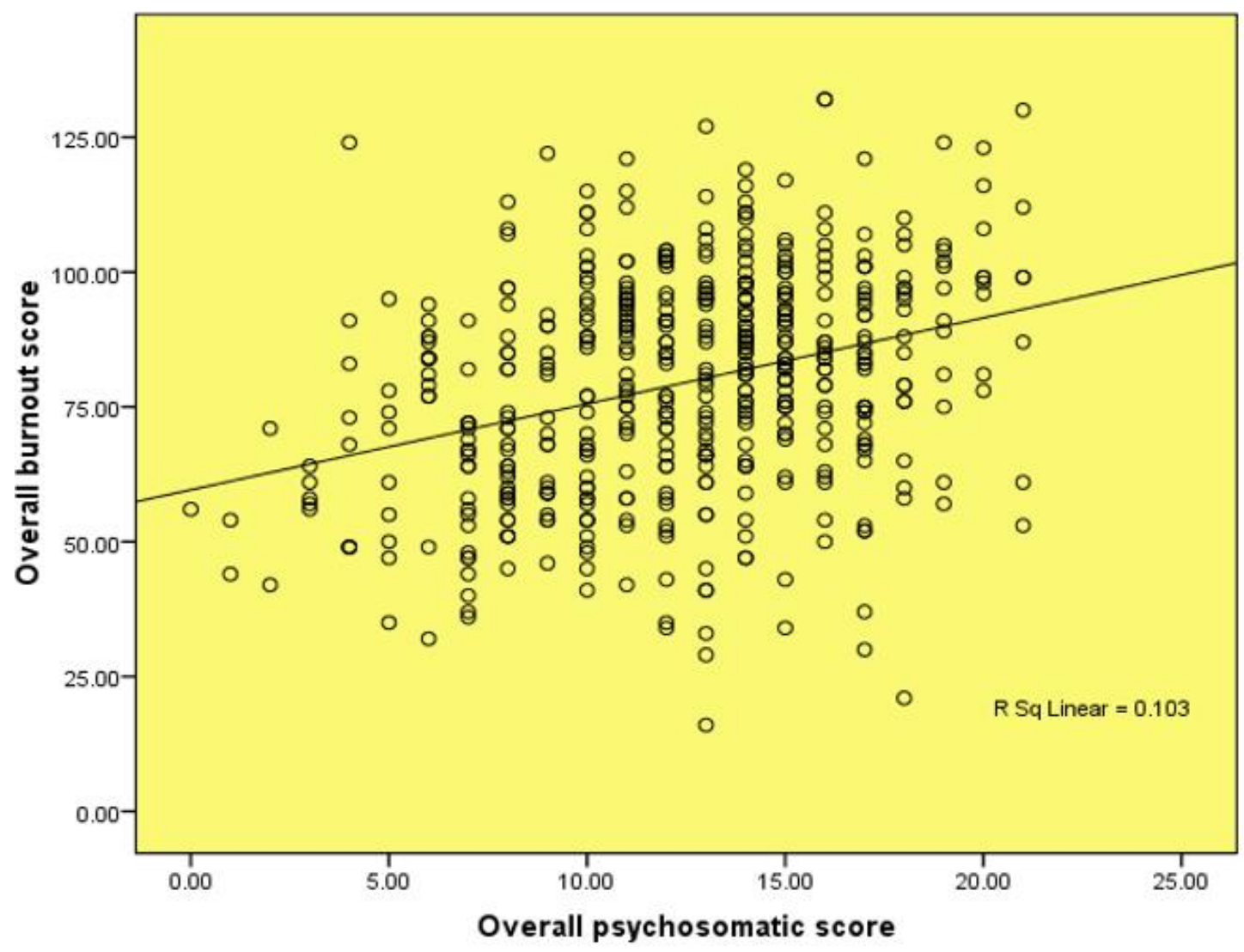

Figure (2): Scatter plot of the relation between overall burnout score and overall psychosomatic score

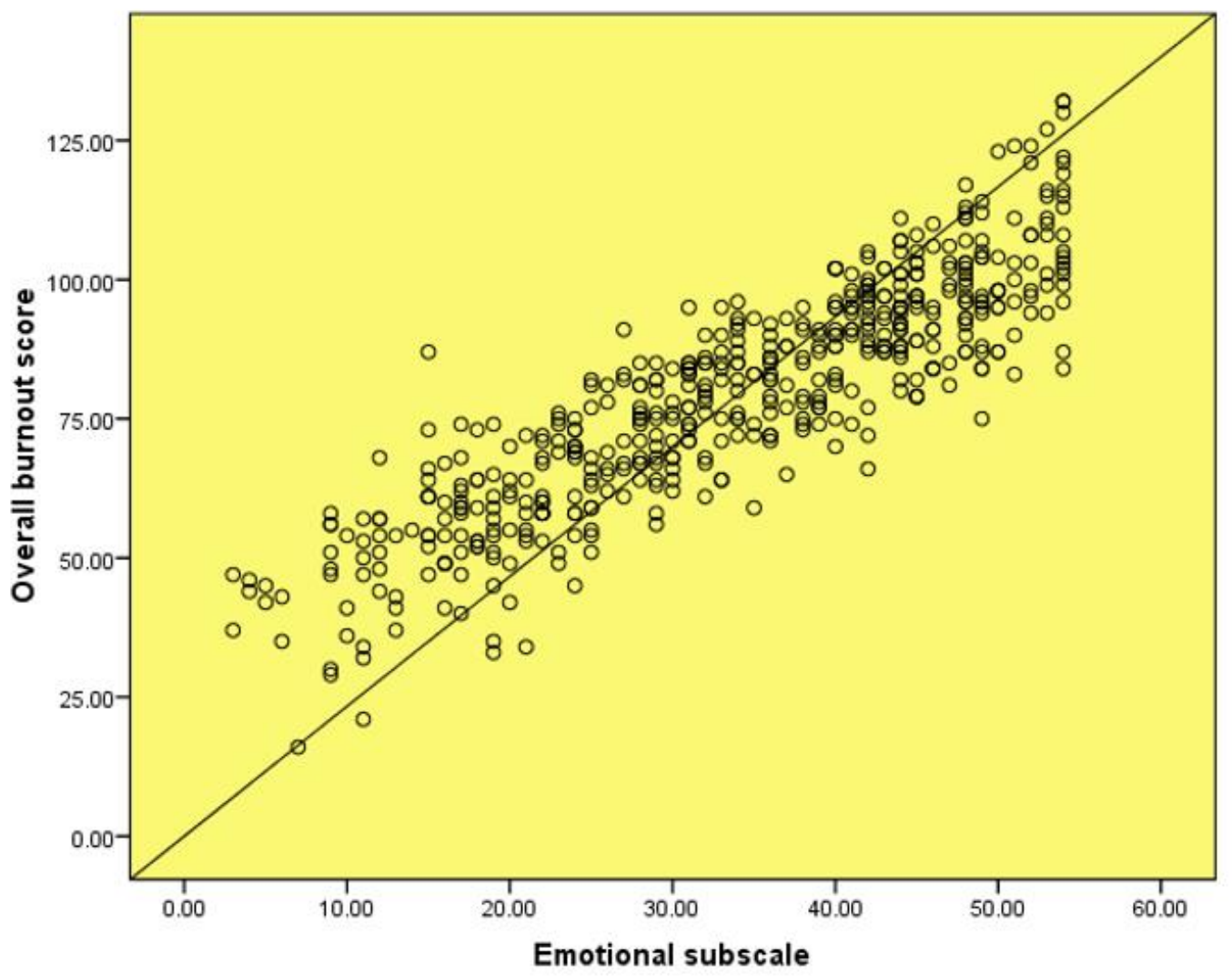

Figure (3): Scatter plot of the relation between overall burnout score and emotional subdomain 


\section{Burnout predictors}

Multivariate linear regression analysis showed that dealing with critical cases, dealing with suspected COVID 19 cases, the number of shifts per month, and the presence of psychosomatic disorders were the significant positive predictors for total burnout score.

Regarding the emotional exhaustion subdomain, dealing with critical cases, the number of shifts per month, and psychosomatic disorders were the significant positive predictors while occupational degree was a significant negative one.

Concerning depersonalization, experience, family size, smoking, dealing with critical cases, the number of shifts per month, and psychosomatic disorders were the significant positive predictors. Family size and experience were significant negative predictors of depersonalization.

The significant predictors for the personal accomplishment domain were; specialty [or the ward where physicians were working], dealing with suspected cases and presence of psychosomatic disorders; while the latter was a negative predictor and others were positive. However, those for the psychosomatic score were sex, residence, chronic disease presence, dealing with critical cases, emotional exhaustion, and low personal accomplishment; while the latter is the negative predictor, others were positive (Table 5).

Table [5]: Multivariate regression analysis for predictors of total burnout and its subscales.

\begin{tabular}{|c|c|c|}
\hline Predictors & $\beta[95 \% C I]$ & p-value \\
\hline Total burnout & \multicolumn{2}{|c|}{$R^{2}=0.169$} \\
\hline Type of the ward & $0.77[0.045-1.5]$ & $0.048 *$ \\
\hline Dealing with critical cases & $2.49[1.28-3.7]$ & $<0.001 * *$ \\
\hline Dealing with suspected cases & $2.55[0.71-4.3]$ & $0.007 * *$ \\
\hline Number of shifts/months & $4.6[0.87-8.3]$ & $0.016^{*}$ \\
\hline Psychosomatic disorders & $1.16[0.74-1.58]$ & $<0.001 * *$ \\
\hline Emotional exhaustion & \multicolumn{2}{|c|}{$R^{2}=0.169$} \\
\hline Occupation & $-2.66[-4.8--0.36]]$ & $0.023 *$ \\
\hline Dealing with critical cases & $1.6[0.94-2.45]$ & $<0.001 * *$ \\
\hline Number of shifts/months & $2.4[0.09-4.7]$ & $0.041^{*}$ \\
\hline Psychosomatic disorders & $0.97[-0.71-1.2]$ & $<0.001 * *$ \\
\hline Depersonalization & \multicolumn{2}{|c|}{$R^{2}=0.137$} \\
\hline Experience & $-1.6[-3.2--0.15]]$ & $0.031 *$ \\
\hline Smoking & $1.22[0.18-2.26]$ & $0.022 *$ \\
\hline Family size & $-1.4[-2.8-0.02]]$ & $0.047 *$ \\
\hline Dealing with critical cases & $0.52[0.092-0.95]$ & $0.017 *$ \\
\hline Number of shifts/months & $1.4[0.08-2.7]$ & $0.037 *$ \\
\hline Psychosomatic disorders & $0.39[0.24-0.54]$ & $<0.001 * *$ \\
\hline Personal accomplishment & \multicolumn{2}{|c|}{$\mathrm{R}^{2}=0.082$} \\
\hline Specialty [Ward] & $0.42[0.083-0.76]$ & $0.016^{*}$ \\
\hline Dealing with suspected cases & $0.92[0.04-1.7]$ & $0.038 *$ \\
\hline Psychosomatic disorders & $-0.20[-0.40-0.014]$ & $0.035^{*}$ \\
\hline Psychosomatic & \multicolumn{2}{|c|}{$R^{2}=0.125$} \\
\hline Sex & $1.07[0.33-1.8]$ & $0.004^{* *}$ \\
\hline Residence & $0.80[0.48-2.33]$ & $0.003 * *$ \\
\hline Presence of chronic disease & $1.40[-2.38-[-0.54]]$ & $0.002 * *$ \\
\hline Dealing with critical cases & $0.27[0.26-0.52]$ & $0.030 *$ \\
\hline Emotional exhaustion & $0.097[0.06-0.13]$ & $<0.001 * *$ \\
\hline Personal Accomplishment & $-0.039[-0.078-0]$ & 0.020 \\
\hline
\end{tabular}

* Significant

** Highly Significant 


\section{DISCUSSION}

Doctor burnout is a worldwide public health problem that is considered an emergency, and the COVID-19 pandemic aggravated it. The COVID-19 pandemic has put a number of physical and enthusiast stressors on the frontline doctors, which has led to an increased doctor burnout ${ }^{(\mathbf{1 3})}$.

The current study was aimed to assess the prevalence of burnout syndrome among frontline physicians working in Egypt's health facilities during the COVID-19 pandemic and identify some of its determinants. The results of this study showed that most of the studied physicians [72.8\%] had a high level of burnout during the COVID-19 pandemic, and a moderate level of burnout was observed in about onequarter [26\%] (Table 1). These results agree with the results of a survey conducted by Medical economics staff, which revealed that more than two-thirds of studied physicians [65\%] reported that the COVID-19 pandemic had increased their feeling of burnout in

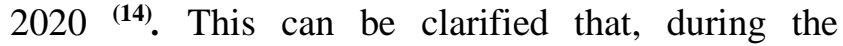
pandemic, doctors have considerable apprehensions of being contaminated with COVID-19 or tainting their families. Notwithstanding increasing workload and longer work hours, PPE deficiencies, absence of assets, and monetary support during the pandemic ${ }^{(15)}$.

In this study, a high level of emotional exhaustion and depersonalization had been detected in more than two-thirds of the studied physicians [62.2\%and62.4\%] respectively, and high levels of reduced personal accomplishment presented in [43.5\%] of the studied participants. This agrees with a similar study conducted in Istituto Auxologico Italiano Hospitals, which found that Moderate to severe levels of emotional exhaustion and reduced personal accomplishment were found in more than $60 \%$ of the studied sample, and moderate to severe levels of depersonalization detected in more than $25 \%$ of the sample during COVID-19 pandemic ${ }^{(16)}$. Emotional exhaustion and depersonalization among frontline health care providers may be mainly due to the COVID-19 pandemic had exacerbated physician's feeling of loss of autonomy as doctors during the pandemic-could be deployed anywhere.

Physicians endured added pressure during the pandemic to pick between their family obligations and their inward feeling of obligation toward patients. Moreover, doctors needed to confront forlornness, the view of disgrace, and inflexible assumptions, which can prompt several enthusiastic and mental results as an outrage, uneasiness, sleep deprivation, and stress.

This study revealed significantly higher scores of burnouts among physicians who were dealing with critical, suspected, and confirmed COVID-19 cases compared to those who weren't $[\mathrm{P}<0.001]$ (Table 3 ). This agrees with an internet survey conducted at Washington University School of Medicine, Barnes Jewish Hospital, and St Louis Children's Hospital on all physician trainees [residents and clinical fellows], and revealed that those who exposed to COVID-19 patients were significantly more stressed and experienced more burnout than those who didn't ${ }^{(17)}$.

This is on the grounds that they confronted a more serious danger of openness to contamination, more workload, ethical problems during care, and need to manage a consistently changing clinical practice climate (e.g., telemedicine supplanting eye to eye experiences, change of protocols for care)

This study revealed that physicians with a higher number of shifts $>10$ per month suffered more burnout (Table 3). This is in line with the study conducted by McHill et al. ${ }^{(18)}$, who reported that lengthy shifts [>80 hr. per week] would almost inevitably result in reduced sleep and rest time, which are well-known triggers of physiological and subjective stress and are potential precursors to burnout.

The current study revealed significantly higher scores of emotional exhaustion among younger age generations of physicians who had lower years of work experience $<10$ compared to those with more years of experience [10-20 and $>20$ years] (Table 2,3). This trend is consistent with other authors' findings showing higher levels of burnout in younger versus older workers, and that burnout is negatively related to years of work experience ${ }^{(\mathbf{1 9})}$. An explanation for this can be fewer younger generations' strategies to cope with the stressors and interact with the work environment. Aging is associated with an increased ability to regulate emotions in the context of reduced perceived lifetime

Smoking, specialty [or the ward where physicians were working], dealing with critical cases, and dealing with suspected cases were the significant predictors of burnout syndrome according to multivariate analysis of variables affecting burnout among the study population (Table 5). This is compared to another study in Italy, which revealed that predictors of all the three burnout components during the COVID-19 outbreak were work hours, psychological comorbidities, fear of infection, and perceived support by friends ${ }^{(2)}$.

Regarding subdomains, occupational degree, dealing with critical cases, and dealing with suspected cases were the significant predictors for emotional exhaustion. The most significant and strongest burnout factor that represents an overwhelming workload and lack of autonomy and control of the work environment is emotional exhaustion.

These study findings revealed that psychosomatic disorders were significant positive predictors for total burnout and its three subscales. And also, emotional exhaustion was a significant predictor for psychosomatic disorders (Table 4,5). This concludes that there is a strong relationship between the emotional and physical condition. In the same context, Salvagioni et al. ${ }^{(20)}$ stated that several 
prospective studies showed physical, psychological, and occupational consequences of job burnout. He stated that burnout was a significant predictor for the many physical consequences like [hypercholesterolemia, type 2 diabetes, coronary heart disease, hospitalization due to cardiovascular disorder, musculoskeletal pain, changes in pain experiences, prolonged fatigue, headaches, gastrointestinal issues, respiratory problems, severe injuries and mortality below the age of 45 years]. And also, for many psychological effects like [insomnia, depressive symptoms, use of psychotropic and antidepressant medications, hospitalization for mental disorders, and psychological ill-health symptoms].

\section{CONCLUSION}

It could be concluded that high degree of burnout and emotional exhaustion prevalence among the Egyptian frontline physicians during the COVID 19 pandemic was higher than those reported internationally and was associated with physical and psychological morbidity and many important job stress sources. This emphasizes the need for preventive interventions and early detection of this health problem in the work environment.

\section{RECOMMENDATIONS}

- Developing an educational intervention programs for health care providers about cope strategies to overcome burnout and job challenges are needed.

- Carry out further research in which burnout causes will be investigated as far as correlation with empathy level is required.

Considering the factors that influencing doctor's satisfaction and professional quality of life is mandatory.

\section{REFERENCES}

1. Sohrabi C, Alsafi Z, O'Neill N, Khan M, Kerwan A, AlJabir A, Losifidis C, Agha R (2020): World Health Organization declares global emergency: A review of the 2019 novel coronavirus [COVID-19]. International Journal of Surgery., DOI: 10.1016/j.ijsu.2020.02.034

2. Giusti E , Pedroli E, D'Aniello $\mathbf{G}$ et al.(2020): The Psychological Impact of the COVID-19 Outbreak on Health Professionals:

ACrossSectionalStudy.FrontiersinPsychology,DOI:https://doi .org/10.3389/fpsyg.2020.01684

3. Unadkat S, Farquhar M (2020): Doctors' wellbeing: selfcare during the covid-19 pandemic. Bmj, DOI: https://doi.org/10.1136/bmj.m1150

4. Satiani B, Zigrang $\mathbf{T}$, Bailey-Wheaton J (2020): COVID19 financial resources for physicians. Journal of vascular surgery. DOI: $10.1016 /$ j.jvs.2020.04.482
5. Lipi R (2020): Doctor, Heal Thyself: Physician Burnout In The Wake Of Covid-19. Forbes. https://www.forbes.com/sites/lipiroy/2020/05/17/doctor-healthyself-physician-burnout-in-the-wake-of-covid19/\#1f3efcd1213c

6. Abdo S , El-Sallamy R, El-Sherbiny A, Kabbash I (2016): Burnout among physicians and nursing staff working in the emergency hospital of Tanta University, Egypt. East Mediterr Health J, 21[12]: 906-915.

7. Wilson W, Raj J, Narayan G, Ghiya M, Murty S, Joseph B (2017): Quantifying burnout among emergency medicine professionals. Journal of emergencies, trauma, and shock, 10[4]:199.

8. Vander Elst T, Cavents C, Daneels K, Johannik K, Baillien E, Van den Broeck A, Godderis L (2016): Job demandsresources predicting burnout and work engagement among Belgian home health care nurses: A cross-sectional study. Nursing outlook, 64[6]: 542-556.

9. World Health Organization (2020): WHO Coronavirus Disease (COVID-19) Dashboard https://covid19.who.int/?gclid=Cj0KCQjwoub3BRC6ARIsA BGhnyapDE4fGBPJKUHxjKKcMtRot0fXnWFWu7xKxCu1c2OcnKf z0Z9_3QaAn4_EALw_wcB

10. Piko B (2006): Burnout, role conflict, job satisfaction and psychosocial health among Hungarian health care staff: A questionnaire survey. Int J Nurs Stud., 43[3]:311-8. DOI:

11. Maslach C, Jackson S, Leiter M (1996): Maslach Burnout Inventory manual. 3rd ed. Palo Alto [CA]: Consulting Psychologist Press. https://www.scirp.org/(S(351jmbntvnsjt1aadkposzje))/referen ce/ReferencesPapers.aspx?ReferenceID=713472

12. Maslach C, Schaufeli W, Leiter M (2001): Job burnout. Annu Rev Psychol. , 52:397-422.

13. Adam S, Mohos A, Kalabay L, Torzsa P (2018): Potential correlates of burnout among general practitioners and residents in Hungary: The significant role of gender, age, dependent care and experience. BMC family practice, 19[1]: 193.

14. Adams J G, Walls R M (2020): Supporting the health care workforce during the COVID-19 global epidemic. Jama., 323[15]: 1439-1440.

15. ALmutairi M , Mahalli A (2020): Burnout and Coping Methods among Emergency Medical Services Professionals. Journal of Multidisciplinary Healthcare, 13: 271.

16. Amol S , Laxmaiah M (2020): The effect of COVID-19 on interventional pain management practices: A physician burnout survey. Pain Physician, 23: S271-S282.

17. Kannampallil T, Goss C, Evanoff B et al. (2020): Exposure to COVID-19 patients increases physician trainee stress and burnout. PloS one, 15(8): e0237301.

18. McHill A , Czeisler C , Shea S (2018): Resident physician extended work hours and burnout. DOI: $10.1093 /$ sleep/zsy112

19. El-Menyar A, Ibrahim W, El Ansari W et al. (2020): Characteristics and predictors of burnout among healthcare professionals: a cross-sectional study in two tertiary hospitals. Postgraduate Medical Journal,DOI: 10.1136/postgradmedj2020-137547

20. Salvagioni D , Melanda F , Mesas A et al.(2017): Physical, psychological and occupational consequences of job burnout: A systematic review of prospective studies. PloS one, 12[10]:e0185781. 